\title{
Prevalencia de Síndrome Metabólico en estudiantes universitarios de Tunja, Boyacá, Colombia, 2014
}

\author{
Lina Fernanda Barrera Sánchez ${ }^{2}$ \\ Juan Manuel Ospina Díaz ${ }^{3}$ \\ Marlon Fernando Tejedor Bonilla4
}

doi:10.11144/Javeriana.ie19-1.psme

Cómo citar: Barrera Sánchez LF, Ospina Díaz JM, Tejedor Bonilla MF. Prevalencia de síndrome metabólico en estudiantes universitarios de Tunja, Boyacá, Colombia, 2014. Investig Enferm. Imagen Desarr. 2017;19(1): 81-93. http://dx.doi.org/10.11144/Javeriana. ie19-1 Javeriana.ie19-1.psme

1. Articulo original de Investigación. Fecha de recepción: 29 abril de 2015. Fecha de aceptación: 13 enero de 2016

2. RN.Magíster Salud Pública. Profesora Asistente Escuela de Enfermería Universidad Pedagógica y Tecnológica de Colombia. Tunja, Boyacá. Investigador Grupo de Investigación en Salud Pública (GISP). Correo electrónico: lina.barrera01@uptc.edu.co.

3. Md.MSc Epidemiología. Profesor Titular Escuela de Medicina Universidad Pedagógica y Tecnológica de Colombia. Tunja, Boyacá. Investigador Grupo de Investigación en Salud Pública (GISP). Correo electrónico: juan.ospina@uptc.edu.co

4. RN. Magíster Salud Pública. Investigador Grupo de Investigación en Salud Pública (GISP). Correo electrónico: surealenfermeria@gmail.com 


\section{Resumen}

En la literatura científica se reporta incremento en la prevalencia de Síndrome Metabólico (SM) en los grupos de escolares y adolescentes, asociado probablemente a modificaciones del estilo de vida, donde se incluyen los hábitos alimenticios y la disminución de la actividad física. Objetivo: Determinar la prevalencia de Síndrome Metabólico y la frecuencia de algunos componentes diagnósticos en una población joven, para aportar una herramienta dirigida a los tomadores de decisiones encaminada a la formulación de políticas públicas de bienestar Universitario. Métodos: Estudio descriptivo de corte transversal. A una muestra de 167 estudiantes universitarios. Se evaluó Índice de Masa Corporal (IMC), perímetro abdominal, además, se realizó determinación de los niveles séricos de Glucosa, High Density Lipoprotein (HDL), Low Density Lipoprotein (LDL), Colesterol total y Triglicéridos (TG), conjuntamente con la presión arterial para determinar la presencia de Síndrome Metabólico. Resultados: Se encontró prevalencia de SM de $8,4 \%$, mayor en hombres $(12,6 \%)$ que en mujeres $(3,75 \%)$. Los componentes diagnósticos que con mayor frecuencia se registraron alterados fueron el aumento del perimetro abdominal $(27,5 \%)$ y la concentración sérica de HDL $(32,7 \%)$. Conclusiones: Se encontró una prevalencia similar a la reportada en la literatura para este grupo de edad. El hallazgo invita a realizar estudios más profundos de tamizaje para diagnóstico precoz e intervención oportuna, así como al diseño de estrategias educativas que faciliten el cambio de estilos de vida en los estudiantes universitarios.

Palabras clave: resistencia a la Insulina; diabetes Mellitus; enfermedades cardiovasculares; adulto Joven; medicina preventiva

\section{Prevalence of Metabolic Syndrome in university students in Tunja, Boyacá, Colombia, 2014}

\section{Abstract}

In the scientific literature it is reported an increase in the prevalence of metabolic syndrome (MS) in groups of schoolchildren and adolescents, probably associated with changes in lifestyle, where eating habits and decreased physical activity are included. Objective: To determine the prevalence of metabolic syndrome and frequency of some diagnostic components in young population, to provide a tool for decision makers aimed at the formulation of public policies for the university welfare. Methods: Crosssectional study. A sample of 167 college students. The Body Mass Index (BMI), waist circumference and also a determination of serum glucose levels was carried out; High Density Lipoprotein (HDL), Low Density Lipoprotein (LDL), total cholesterol and triglycerides (TG), were also gauged in conjunction with the blood pressure to determine the presence of metabolic syndrome. Results: prevalence of MS was found $8.4 \%$ higher in men $(12.6 \%)$ than women $(3.75 \%)$. the diagnostic components which were most frequently reported were increased abdominal circumference $(27.5 \%)$ and serum HDL concentration (32.7\%). Conclusions: a similar prevalence of that reported in the literature for this age group was found. The discovery invites us to make further study of screening for early diagnosis and intervention, as well as the design of educational strategies to facilitate the change of lifestyle in college students.

Keywords: Insulin resistance; Mellitus diabetes; cardiovascular diseases; Young adult; preventive medicine 


\section{Prevalência da Síndrome Metabólica em estudantes universitários de Tunja, Boyacá, Colômbia, 2014}

\section{Resumo}

Na literatura científica se relata incremento na prevalência da Sindrome Metabólica (SM) nas turmas de escolares e adolescentes, associado a prováveis alterações no estilo de vida, onde estão incluídos hábitos alimentares e diminuição de atividade física. Objetivo: Determinar a prevalência da Sindrome Metabólica e a frequência de alguns componentes diagnósticos em uma população jovem, para fornecer ferramenta dirigida a tomadores de decisões, voltada para a formulação de políticas públicas de bem-estar universitário. Métodos: Estudo descritivo transversal sobre amostra de 167 estudantes universitários. Foi avaliado Índice de Massa Corporal (IMC), circunferência abdominal, foi realizada determinação de níveis em soro de Glucose, High Density Lipoprotein (HDL), Low Density Lipoprotein (LDL), Colesterol total e Triglicérides (TG), em conjunto com pressão arterial para determinar a presença de Síndrome Metabólica. Resultados: Encontrou-se prevalência de SM de 8,4\%, maior em homens $(12,6 \%)$ do que mulheres $(3,75 \%)$. Os componentes diagnósticos que com mais frequência apareceram alterados foram o aumento da circunferência abdominal $(27,5 \%)$ e a concentração em soro de HDL (32,7\%). Conclusões: Foi encontrada prevalência semelhante à relatada na literatura para esta faixa etária. O achado convida aprofundar em estudos de tamisação para diagnóstico precoce e intervenção oportuna, bem como a criar estratégias educacionais que facilitem a mudança de estilos de vida em estudantes universitários.

Palavras-chave: resistência à Insulina; diabetes Mellitus; doenças cardiovasculares; adulto Jovem; medicina preventiva 


\section{Introducción}

En los países emergentes, la transición epidemiológica se evidencia con un inusual incremento de enfermedades crónicas; en particular diabetes y enfermedad cardiovascular, patologías significativas en mayores de cuarenta años. Se adelantan esfuerzos por identificar indicadores de pronóstico o predictores en las poblaciones más jóvenes; por ejemplo, patrones dietarios, actividad física, consumo de alcohol y tabaquismo.

En Colombia, la prevalencia de Diabetes Mellitus tipo 2 (DM2) varía entre $4 \%$ y $8 \%$, siendo menor en las zonas rurales (2\%), de lo cual se desprende la importancia de factores ligados a los procesos sociales y económicos de urbanización, como dieta rica en calorías y disminución de la actividad física que pueden generar obesidad, la cual ocurre en más del 30\% de los habitantes urbanos y Sindrome Metabólico presente en el 20-35\% de la población (1). La intolerancia a la Glucosa es casi tan frecuente como la DM2. En 20052006, se adelantó una investigación en universitarios que registró prevalencia de glicemia en ayunas $\geq 100 \mathrm{mg} / \mathrm{dl}$, en $8.4 \%$, además de intolerancia a la Glucosa de $0.8 \%$ (1). Según el análisis de situación de salud para Colombia 2014 las enfermedades hipertensivas fueron responsables del 9,35\% (22.476) de los decesos por enfermedades del sistema circulatorio en hombres, equivaliendo al 4,82\% del total de muertes en el grupo de causas. En mujeres, estás fueron responsables del 11,02\% (24.941) de los decesos por enfermedades del sistema circulatorio y ocasionaron el 5,34\% del total de muertes. En cuanto a Diabetes Mellitus causó el 12,73\% (24.097) de los decesos en el grupo de hombres, equivalente al 6,36\% del total de muertes en el grupo de las demás causas, y el 16,91\% (32.058) de los decesos en el grupo en mujeres, equivalente al $8,46 \%$ del total de muertes en el grupo de las demás causas (2).

El Síndrome metabólico, se define como un conjunto de factores de riesgo para Diabetes Mellitus tipo 2 y enfermedad cardiovascular, caracterizado por la presencia de Resistencia a la Insulina (RI) e Hiperinsulinismo compensador. Condiciones asociadas a trastornos del metabolismo de los carbohidratos y los lipidos. Gerald Reaven, de la Universidad de Stanford, describió un grupo de factores de riego a los que llamó Síndrome X, en razón a que estos factores: dislipidemia, hipertensión arterial e hiperglicemia, cursaban juntos en un apreciable número de pacientes (3). Posteriormente la Organización Mundial de la Salud (OMS) acuñó en 1998 el término Síndrome Metabólico y definió unos criterios para caracterizarlo (4).

El SM es el producto de un proceso interaccionante de anormalidades del endotelio vascular; estrés oxidativo; grasa visceral que genera síntesis de adipocitoquinas inflamatorias y sintesis y liberación de Cortisol, que ocurren la mayoría de las veces en un entorno fisiológico facilitado por la obesidad y la creciente resistencia a la Insulina, bajo el influjo de una predisposición genética y características étnicas particulares (5).

Desde 1999, la OMS ha manifestado que el interés científico debe centrarse en una característica metabólica muy llamativa en el SM, que es la Resistencia a la Insulina. En base a este concepto la National 
y posteriormente la International Diabetes Federation (IDF) se han dado a la tarea, desde 1995, de estructurar una serie de criterios diagnósticos que sean fácilmente aplicables, sensibles y específicos para el diagnóstico temprano del SM (6).

La IDFha establecido los criterios diagnósticos del SM $\left({ }^{7}\right)$. El Consenso Colombiano de Síndrome Metabólico acogió los criterios de la IDF para el diagnóstico $\left({ }^{8}\right)$. Se considera que existe Síndrome Metabólico si al aumento del Perimetro Abdominal (PA) se agregan dos de los siguientes criterios: Triglicéridos séricos por encima de $150 \mathrm{mg} / \mathrm{dl}$; Colesterol HDL por debajo de $40 \mathrm{mg} / \mathrm{dl}$ en los varones o de $50 \mathrm{mg} / \mathrm{dl}$ en las mujeres; presión arterial diastólica por encima de $85 \mathrm{mmHg}$ y/o presión sistólica por encima de 130 mmHg: Glucosa en plasma superior a $100 \mathrm{mg} / \mathrm{dl}$ y/o Diabetes Mellitus ya diagnosticada (8).

El SM incrementa hasta dos veces el riesgo de enfermedad cardiovascular y hasta cinco la probabilidad de DM2. El desarrollo tecnológico y la industrialización han hecho la vida más confortable y sedentaria, lo que unido a inadecuados hábitos dietéticos y predisposición genética, favorece el incremento de la prevalencia de SM. El criterio diagnóstico que más se utiliza es el propuesto por la NCEP-ATP III (9).

Los factores de riesgo cardiovascular se agrupan en tres categorias: generales, conductuales e intermedios. Todos ellos están ligados a enfermedad coronaria y aterogénesis, y son condicionados por variables socioambientales y comportamientos adquiridos desde edades tempranas del desarrollo (10).

En Colombia, en 2012 se evidencia que el grupo de 14-26 años no reporta enfermedades cardiovasculares entre las primeras causas de morbimortalidad; no obstante, la Hipertensión arterial fue un diagnóstico muy frecuente en el grupo de 27-59 años en consulta en 2011: 10,4\% mujeres y $8,7 \%$ hombres (11), incluida la población universitaria (12).

Evidencia de SM en jóvenes, se reconoce como un significativo predictor de enfermedades cardiovasculares y DM2 (13). Según la encuesta Nacional de Situación Nutricional en Colombia (ENSIN 2010) el 16.7\% de jóvenes de 10 a 17 años presentaron exceso de peso, el 3,4\% obesidad (siendo las mujeres más afectadas por el sobrepeso y los hombres por la obesidad). Se halló mayor exceso de peso en la zona urbana con un 19,2\%, frente al área rural con un $13,4 \%$. Las ciudades capitales presentaron mayor proporción de jóvenes con exceso de peso. La prevalencia de obesidad abdominal medida por la circunferencia de la cintura a nivel nacional fue de $39,8 \%$ para hombres y $62 \%$ para mujeres de 18 a 64 años de edad (14).

No se conocen estudios que establezcan la prevalencia de Síndrome Metabólico en Boyacá en grupos de estudiantes universitarios que revelen la magnitud del problema.

El estudio tuvo como objetivo determinar la prevalencia de SM y frecuencia de algunos componentes diagnósticos del sindrome en una población joven, para aportar a quienes toman decisiones, una herramienta en la formulación de políticas públicas encaminadas al bienestar Universitario. 


\section{Métodos}

Se diseñó un estudio de corte transversal, se definió como población de estudio a los estudiantes universitarios. La población objetivo fueron 17.000 estudiantes matriculados en una universidad pública. Con base en la prevalencia de SM de 4,6\% reportada en un estudio similar (15), se estimó un tamaño de muestra mínimo de 134 sujetos, con $a=0,05$, diferencias mínimas de $5 \%$ y efecto de diseño=2. Mediante muestreo secuencial no probabilístico se obtuvo la participación de un total de 167 personas. La muestra se seleccionó con previo consentimiento informado de los estudiantes que asistían a la práctica de un test serología (VDRL), programado por la universidad como requisito de matrícula y que cada estudiante debe realizarse anualmente. Esta norma institucional permitió facilitar la obtención de una muestra de suero para la evaluación bioquímica de lípidos y Glucosa en ayunas.

Mediante entrevista directa se aplicaba una encuesta para obtener información de tipo sociodemográfico. Se incluyeron en la base de datos quienes aceptaron participar en el estudio y aportaron la información y alícuota de suero requerida. Se excluyeron quienes no se encontraban en ayunas o no aceptaron participar.

\section{Instrumentos y recolección de la información}

En la antropometría se empleó una balanza de pie calibrada con anterioridad, tallímetro Kramer; cinta métrica no distensible de fijación automática (SECA), tensiómetro. El análisis de sueros fue realizado de manera enmascarada, por bacterióloga entrenada, quien solo conoció un código para cada muestra. Mediante entrevista, se indago sobre aspectos sociodemográficos. El diagnóstico se ciñó a los criterios de la IDF para SM: positivo con la presencia de 3 de 5 criterios: Perímetro Abdominal $>90 \mathrm{~cm}$ para los hombres y $>80 \mathrm{~cm}$ para las mujeres, TG $>150 \mathrm{mg} / \mathrm{dl}, \mathrm{HDL}<40 \mathrm{mg} / \mathrm{dl}$ para hombres y $<50 \mathrm{mg} / \mathrm{dl}$ para hombres, valores de glicemia en sangre en ayunas iguales o superiores a $100 \mathrm{mg} / \mathrm{dl}$, cifras de presión arterial sistólica $\geq 130 \mathrm{mmHg}$ y/o presión arterial diastólica $\geq 85 \mathrm{mmHg}$, diagnóstico previo de Diabetes tipo 2. Eventualmente se consideró el valor del Índice de Masa Corporal igual o superior a 25 como criterio de sobrepeso u obesidad.

Con previo ayuno de mínimo 8 horas de todos los participantes, se extrajeron mediante venopunción, 5 cc de sangre periférica, en tubos de laboratorio secos vacutainer. Una vez retraído el coágulo se procedió a centrifugar durante 10 minutos a $3.000 \mathrm{rpm}$ hasta obtener suero. La determinación de los niveles séricos de Glucosa y perfil lipídico se adelantó en el equipo Microlab 100 (Merck®), mediante técnica colorimétrica inmunoenzimática.

\section{Plan de Procesamiento y análisis de los datos}

Se utilizó el programa Epi-Info 2002. El Análisis Univariado permitió examinar los dos grupos (con SM o sin él) y determinar su comparabilidad. Cada 
variable fue descrita en su nivel; medias para las variables continuas, proporciones para las categóricas o nominales. Todas se reportaron con sus Intervalos de Confianza (IC) a nivel alfa de 0.05. Cuando fue necesaria la comparación de grupos en variables cuantitativas, se utilizó la t de Student para muestras independientes.

Con al Análisis Bivariado se buscó establecer asociación de las covariables con la variable de salida SM, calculando medidas de efecto absoluto (16); se estimó la Odss Ratio (OR) dada la exposición e IC al 95\%, además del valor p (17). Se realizó análisis estratificado para control de sesgos.

\section{Aspectos éticos}

Este estudio fue considerado investigación de riesgo mínimo acorde con la resolución 8430 del Ministerio de Salud (18). Se tomaron muestras para pruebas serológicas de diagnóstico; por lo tanto, se solicitó un consentimiento informado por escrito a las personas que aceptaron participar. Durante y después del proceso de ejecución se respetaron los principios éticos de beneficencia, no maleficencia, autonomía y justicia (19).

\section{Resultados}

En un total de 167 estudiantes, 80 son mujeres (48\%) y 87 hombres (52\%). La edad media fue de 20,9 años (SD=3,06; Rango: 16-33 años). Con respecto al estrato socioeconómico $44,9 \%(n=75)$ pertenece al estrato $2 ; 31,7 \%(n=53)$ al 3; el $15,6 \%(n=26)$ al 1 y el $7,8 \%(n=13)$ al 4 .

La evaluación del Índice de Masa corporal muestra que 11,4\% ( $n=19)$ se encuentra en bajo peso; $73 \%(n=122)$ están en rango de normalidad; $15,5 \%$ $(n=26)$ tienen sobre peso y el 2,4 $(n=4)$ en sobre peso grado II. $26,9 \%$ de los que se encuentran en sobre peso cumplen criterios para ser diagnosticados como SM. $28,1 \%(\mathrm{n}=47)$ presenta PA por encima de lo normal.

El 12,5\% ( $n=21)$ de la muestra registró hipertensión con valores iguales o por encima de 130/85 mmHG.El análisis del Colesterol evidencia que el $23,75 \%(n=19)$ de las mujeres y el 20,7\% $(n=18)$ de los hombres tienen valores superiores a $200 \mathrm{mg} / \mathrm{dl}$, no siendo significativa esta diferencia ( $\mathrm{p}=0,38)$. En conjunto, 22,16\% de los universitarios registra anormalidad en los niveles séricos de HDL. El 24\% (n=40) de los estudiantes tienen valores de triglicéridos iguales o superiores a $150 \mathrm{mg} / \mathrm{dl}$, y el $0,6 \%(\mathrm{n}=1)$ tiene glicemia basal superior a $100 \mathrm{mg} / \mathrm{dl}$ (Tabla 2).

Acorde con los criterios diagnósticos de la IDF sobre el SM, la prevalencia de SM en la muestra estudiada es 8,4\%; el análisis evidencia que el $36 \%(n=29)$ de las mujeres y el 20,7\% $(n=18)$ de los hombres presentan valores de perímetro abdominal por encima de lo normal para latinoamericanos, siendo esta diferencia estadisticamente significativa $(p=0,025)$. Es importante anotar, que de los criterios IDF establecidos para el diagnóstico de SM, el perímetro abdominal aumentado es el componente que con mayor frecuencia se encuentra en el total de participantes y en las mujeres, mientras que en los hombres, las concentraciones séricas de Triglicéridos predominaron. 
De igual manera, se encuentra que estos criterios se acompañaron más veces por el aumento de Triglicéridos y HDL bajo en las mujeres y por aumento del PA y HDL bajo en los hombres.

Tabla 1. Prevalencia total y por sexo, del Síndrome Metabólico según los Criterios diagnósticos de la IDF. Fuente: base de datos.

\begin{tabular}{|c|c|c|c|c|c|c|c|c|}
\hline \multirow{2}{*}{\multicolumn{2}{|c|}{ Variable }} & \multicolumn{2}{|c|}{ HOMBRE } & \multicolumn{2}{|c|}{ MUJER } & \multicolumn{2}{|c|}{ TOTAL } & \multirow{2}{*}{$\mathbf{P}$} \\
\hline & & \multirow{2}{*}{$\begin{array}{c}\mathbf{N}^{\circ} \\
18 \\
\end{array}$} & \multirow{2}{*}{$\begin{array}{c}\% \\
20,68 \\
\end{array}$} & \multirow{2}{*}{\begin{tabular}{|c|}
$\mathbf{N}^{\circ}$ \\
29 \\
\end{tabular}} & \multirow{2}{*}{\begin{tabular}{|c|}
$\%$ \\
36,25 \\
\end{tabular}} & \multirow{2}{*}{\begin{tabular}{|c|}
$\mathbf{N}^{\circ}$ \\
47 \\
\end{tabular}} & \multirow{2}{*}{\begin{tabular}{|c|}
$\%$ \\
28,14 \\
\end{tabular}} & \\
\hline Perimetro & Presente & & & & & & & \multirow{2}{*}{0,039} \\
\hline Abdominal & Ausente & 69 & 79,32 & 51 & 63,75 & 120 & 71,86 & \\
\hline \multirow{2}{*}{ HTA } & Presente & 4 & 4,59 & 0 & 0 & 4 & 2,39 & \multirow{2}{*}{0,31} \\
\hline & Ausente & 83 & 95,41 & 80 & 100,0 & 163 & 97,61 & \\
\hline \multirow{2}{*}{ HDL } & Presente & 18 & 20,68 & 10 & 12,5 & 28 & 16,76 & \multirow{2}{*}{0,22} \\
\hline & Ausente & 69 & 79,32 & 70 & 87,5 & 139 & 83,24 & \\
\hline \multirow{2}{*}{ Triglicéridos } & \begin{tabular}{|l|} 
Presente \\
\end{tabular} & 27 & 31,0 & 13 & 16,3 & 40 & 24,0 & \multirow{2}{*}{0,039} \\
\hline & Ausente & 60 & 69,0 & 67 & 83,7 & 127 & 76,0 & \\
\hline \multirow{2}{*}{$\begin{array}{l}\text { Glicemia } \\
\text { Basal }\end{array}$} & Presente & 0 & 0,0 & 1 & 1,25 & 1 & 0,59 & \multirow{2}{*}{0,51} \\
\hline & Ausente & 87 & 100,0 & 79 & 98,75 & 166 & 99,41 & \\
\hline \multirow{2}{*}{ SM por sexo } & Presente & 11 & 12,64 & 3 & 3,75 & 14 & 8,38 & \multirow{2}{*}{0,07} \\
\hline & Ausente & 76 & 87,36 & 77 & 96,25 & 153 & 91,62 & \\
\hline
\end{tabular}

Fuente: Elaboración propia.

TABLA 2. Evaluación de asociación de algunos factores con el SM. Fuente: Base de datos

\begin{tabular}{|c|c|c|c|c|c|c|}
\hline \multirow{2}{*}{ FACTOR } & \multirow{2}{*}{ VALOR } & \multicolumn{2}{|c|}{$\begin{array}{c}\text { SINDROME } \\
\text { METABOLICO }\end{array}$} & \multicolumn{3}{|c|}{ ASOCIACION } \\
\hline & & SI & No & OR & IC95\% & $\mathbf{p}$ \\
\hline \multirow{2}{*}{ SEXO } & HOMBRE & 11 & 76 & \multirow{2}{*}{3,71} & \multirow{2}{*}{$0,99-13,8$} & 0,034 \\
\hline & MUJER & 3 & 77 & & & \\
\hline \multirow{2}{*}{ IMC } & $>25$ & 10 & 16 & \multirow{2}{*}{21,4} & \multirow{2}{*}{$6,0-76,2$} & $<0,0001$ \\
\hline & $\leq 25$ & 4 & 137 & & & \\
\hline \multirow{2}{*}{ FUMADOR } & SI & 4 & 41 & \multirow{2}{*}{1,09} & \multirow{2}{*}{$0,3-3,6$} & 0,55 \\
\hline & NO & 10 & 112 & & & \\
\hline \multirow{2}{*}{ ANTEC DIABETES } & SI & 8 & 67 & \multirow{2}{*}{1,71} & \multirow{2}{*}{$0,5-5,17$} & 0,24 \\
\hline & NO & 6 & 86 & & & \\
\hline \multirow{2}{*}{ ANTEC ENF CV } & SI & 4 & 23 & 2,26 & \multirow{2}{*}{$0,65-7,8$} & 0,23 \\
\hline & NO & 10 & 130 & & & \\
\hline \multirow{2}{*}{ CONSUME MEDIC } & SI & 2 & 10 & 2,38 & \multirow{2}{*}{$0,46-12,1$} & 0,26 \\
\hline & NO & 12 & 143 & & & \\
\hline CONSUME CAFÉ & SI & 10 & 91 & 1,7 & $0,5-5,6$ & 0,28 \\
\hline
\end{tabular}

Fuente: Elaboración propia. 
La prevalencia de SM en el presente estudio de investigación es mayor en hombres con un $12,6 \%(n=11)$, que en mujeres con un $3,75 \%(n=3)$. Aunque la diferencia no es significativa $(\mathrm{p}>0,05)$, la probabilidad de desarrollar SM es ligeramente mayor en los hombres (Tabla 1).

De manera exploratoria se buscó identificar algunos factores relacionados con el diagnóstico de SM. El sexo masculino y un IMC aumentado se encontraron significativamente relacionados, otras variables que se asocian al SM, aunque de manera no significativa fueron los antecedentes de diabetes o enfermedad cardiovascular, así como el consumo de café y la medicación (Tabla 2).

Teniendo en cuenta que el estilo de vida y algunos factores biológicos inciden sobre el riesgo de manifestación del SM, se realizó una exploración cuantitativa de la presencia de algunos de ellos, en la tabla 2 se presentan algunas asociaciones que se encontraron significativamente asociadas con el SM, y otras que no siendo significativas, podrian ser objeto de estudio con muestras de mayor tamaño.

\section{Discusión}

La combinación de criterios antropométricos con datos bioquímicos proporciona al esquema diagnóstico del SM, un alto grado de solidez en términos de validez y reproducibilidad, lo que amerita considerar los datos como un indicio concreto que invita a estudiar con más profundidad el SM, habida cuenta de su condición problema de Salud Pública.

La prevalencia estimada en este trabajo es cercana a la reportada en un estudio similar adelantado en Paraguay con adolescentes en sobrepeso (20). También es bastante similar a lo reportado recientemente por Ruano y colaboradores en Ecuador en un estudio realizado también en estudiantes universitarios (21). Por otra parte e ha observado que la prevalencia de SM se incrementa con la edad, pues en una evaluación adelantada en Estados Unidos se encontró está condición en un 6,9\% en el grupo de 20 a 29 años, cifra que llegó a 43,5\% en el grupo de 60-69 años (22). Además, es importante considerar que de manera global se percibe una tendencia hacia el incremento (23). No resulta fortuito que la Organización Mundial de la Salud declarase a la obesidad como la primera epidemia ocasionada por una enfermedad no transmisible (24).

La obesidad abdominal, expresada como un aumento significativo del perimetro abdominal parece ser el principal marcador de la probabilidad de que un adolescente o adulto joven presente SM, teniendo en cuenta que es el criterio que con mayor frecuencia ocurre entre quienes cumplen los criterios diagnósticos del SM (25). Por esta razón, se considera fundamental que en los servicios de salud se retome la práctica de mediciones antropométricas como parte de la rutina en la atención directa de los pacientes adolescentes.

Los niveles séricos de Triglicéridos y el aumento del PA fueron los criterios diagnósticos que con mayor frecuencia ocurrieron en la muestra estudiada: $32,7 \%$ y $27,5 \%$ respectivamente, lo que lleva a pensar en considerarlos 
unos buenos marcadores o predictores del SM. Es importante anotar que el primero de estos dos criterios se observó con mayor frecuencia en las mujeres, hallazgo similar a lo reportado por un estudio adelantado por Fernández y colaboradores en España (26). Aunque no se realizó evaluación en este sentido, es importante anotar que algunos estudios han sugerido cierta relación del SM con variables sociodemográficas, entre las que destacan el estrés crónico: condición relativamente frecuente entre los estudiantes universitarios (27). También es importante considerar otros factores ambientales y culturales que pueden favorecer la ganancia excesiva de peso desde edades muy tempranas, tal es el caso del descenso marcado en los niveles de actividad física que suelen desarrollar los estudiantes universitarios y que gradualmente son abandonados para entrar en una condición de marcado sedentarismo. Además de la tendencia a consumir alimentos y bebidas altamente cargados de azucares y carbohidratos omitiendo el consumo de alimentos ricos en fibra (28).

En lo que respecta a los niveles de HDL, el resultado de menores niveles en mujeres concuerda parcialmente con lo hallado en un estudio adelantado en China, en el que se observó mayor correlación entre bajos niveles de HDL y SM en los hombres que en las mujeres (29).

Un aspecto que falta definir mejor del SM en jóvenes, tiene que ver con la acumulación excesiva de grasa abdominal, evento que se ha reportado asociado con ateroesclerosis y consecuentes daños cardiovasculares, toda vez que citoquinas pro-inflamatorias son secretadas desde los tejidos adiposos viscerales. El estudio de Hyun Kyung Bae, et al. sugiere que en los jóvenes afectados con SM es elevada la probabilidad de que estén cursando con afectación directa del Ventrículo izquierdo (30).

La significativa prevalencia encontrada en este estudio exploratorio, obliga a llamar la atención, sobre el impacto potencial que podría representar a futuro la progresión de este síndrome a la categoría de Diabetes Mellitus y/o enfermedad cardiovascular franca. De esta consideración surge la urgencia de proponer estrategias de prevención a través de las acciones de búsqueda activa, detección precoz e intervención oportuna de casos y el fomento de cambios comportamentales en el estilo de vida de jóvenes y adolescentes susceptibles. En este sentido se han manifestado varios investigadores dada la fuerte preocupación en torno a las dificultades que se plantean en términos de adherencia al tratamiento y logro de control metabólico del paciente diabético ya diagnosticado (31).

Como limitaciones del estudio cabría mencionar que para obtener mayor significancia sería conveniente adelantar más investigaciones, con muestras mayores y criterios de selección que permitan una aleatorización completa. No obstante, se considera que el trabajo adelantado constituye una primera aproximación hacia el abordaje de un problema de Salud Pública no explorado hasta el momento en la comunidad universitaria de Boyacá.

\section{Conclusiones}


y en mujeres fue el aumento del perímetro abdominal, mientras que en los hombres ocurrió con mayor frecuencia la alteración de los niveles séricos de Triglicéridos, lo cual invita a incrementar los esfuerzos para desarrollar estrategias de búsqueda activa de pacientes afectados, así como a la implementación de metodologías de educación y fomento de la salud que faciliten que los estudiantes universitarios adopten estilos de vida saludables.

\section{Financiación}

El presente estudio se desarrolló como parte final del proceso de formación de un Maestrante en Salud Pública y se financió con recursos propios del Grupo de Investigación en Salud Pública (GISP).

\section{Conflicto de Intereses}

Ninguno

\section{Agradecimientos}

Los autores hacen expresa manifestación de reconocimiento y gratitud al Grupo de Investigación en Salud Pública por el apoyo académico y logístico, también a los estudiantes que amablemente y de manera desinteresada, accedieron participar en esta investigación.

\section{Referencias}

1. Feliciano-Alfonso JE, Mendivil CO, Ariza IDS, Pérez CE. Cardiovascular risk factors and metabolic syndrome in a population of young students from the National University of Colombia. Rev Asso Medica Bras. 2010;56(3):293-8

2. República de Colombia. Ministerio de Protección Social. Análisis de Situación de Salud.169 pgs. [Internet] 2014. Disponible en: http:// www.minsalud.gov.co/sites/rid/Lists/BibliotecaDigital/RIDE/VS/ED/ PSP/ASIS_2014_v11.pdf

3. Pineda, CA. Síndrome metabólico: definición, historia, criterios. Colom. Med.. 2008; 39(1): 96-106.

4. Alberti KG, Zimmet PZ. Definition, diagnosis and classification of diabetes mellitus and its complications. Part 1: diagnosis and classification of diabetes mellitus provisional report of a WHO consultation. Diabet Med. 1998; 15: 539-53.

5. Pajuelo J, Sánchez-Abanto J, Torres HL, Miranda M. Prevalencia del síndrome metabólico en pobladores peruanos por debajo de $1000 \mathrm{y}$ por encima de los 3000 msnm. An Fac Med. 2012; 73(2): 101-106.

6. Bermúdez P, Balmore J, Finol G, Fredy J, Leal N, Parra MG, et al. Prevalencia del síndrome metabólico en la población adulta Añu de la laguna de Sinamaica del municipio de Páez, Estado Zulia. Rev Latinoam Hiperte. 2009; 4(3): 64-70.

7. International diabetes federation. The IDF consensus worldwide definition of the Metabolic Syndrome. Bruselas, 2006.

8. Asociación Colombiana de Endocrinología. Consenso Colombiano de síndrome metabólico. Bogotá; 2006. p. 26. 
9. Bojorges LA, Castillo J A, Jiménez R. Factores de riesgo de síndrome metabólico en estudiantes de la universidad Pablo Guardado Chávez, año 2013. Rev Cubana Invest Bioméd. [Internet] 2013 Dic [Citado en 09 de Mayo de 2014]; 32(4): 379-388 Disponible en: http://scielo.sld.cu/ scielo.php?script=sci_arttext\&pid=S086403002013000400001\&lng=es.

10. Alayón AN, Ariza S, Baena K, Lambis L, Martínez L, Benítez L. Búsqueda activa y evaluación de factores de riesgo cardiovascular en adultos jóvenes, Cartagena de Indias, 2007. Biomédica. 2010;30(2): 38-44

11. olombia. Ministerio de Salud y Protección Social. Análisis de situación de salud de poblaciones diferenciales. Grupo ASIS. Bogotá D.C. 2012.

12. Ledo-Varela M, De Luis-Román A, González-Sagrado M, Izaola O, Conde $\mathrm{R}$, et al. Características nutricionales y estilo de vida en universitarios. Rev Nutr Hosp. 2011; 26(4):814-818.

13. Trejo-Gutiérrez, JF. Epidemiología del Síndrome Metabólico y Diabetes mellitus tipo 2. ¿El diluvio que viene? Archivos de Cardiología de México. 2004; 74(S2):S267-S270.

14. Colombia. Ministerio de la Protección social. ICBF. PROFAMILIA. INS. Encuesta Nacional de la situación Nutricional en Colombia. [Internet] 2010. Disponible en: http://www.icbf.gov.co/portal/page/portal/ PortalICBF/Bienestar/ENSIN1/ENSIN2010/LibroENSIN2010.pdf.

15. Maiz G. A. El Síndrome metabólico y riesgo cardiovascular. Boletín de la Escuela de Medicina. 2005; 30(1): 25-30.

16. Kelsey J1, Douglas W, Evans AS. Methods in observational epidemiology. New York: Oxford University Press. 1986.

17. Greenland S. Modelling and variable selection in epidemiologic analysis.Am J Public Health. 1989; 79:340-9.

18. Colombia. Ministerio de Salud de Colombia. Resolución 8430 de 1993. Por la cual se establecen las normas científicas, técnicas y administrativas para la investigación de salud. Bogotá D.C. 1993.

19. Coughlin S, Beauchamp TL. Confidentiality and privacy protection in epidemiologic research. Ethics and Epidemiology; Oxford University Press, New York. 1996.

20. López P, Araujo C, Leguizamón C, Ayala A, Scott C, Maldonado D. Prevalencia de sindrome metabólico en adolescentes con sobrepeso $\mathrm{u}$ obesidad. Pediatr (Asunción). 2012; 39(1): 21-25

21. Ruano CI, Melo JD, Mogrovejo L, de Paula KR, Espinoza CV. Prevalencia de síndrome metabólico y factores de riesgo asociados en jóvenes universitários. Nutr Hosp. 2015;31(4):1574-1581

22. Villegas A, Botero JF, Arango IC, Arias S, Toro MM. Prevalencia del Síndrome metabólico en El Retiro, Antioquia. Iatreia. 2003; 16(4): 291-297.

23. Hedley AA, Ogden CL, Johnson CL, Carroll MD, Curtin LR, Flegal KL. Prevalence of Overweight and Obesity Among US Children, Adolescents, and Adults, 1999-2002. JAMA. [Internet] 2004; 291(23): 2847-2850. Disponible en: http://doi:10.1001/jama.291.23.2847. 
24. Szer G, Kovalskys I, De Gregorio MJ. Prevalencia de sobrepeso, obesidad y su relación con hipertensión arterial y centralización del tejido adiposo en escolares. Arch Argent Pediatr. 2010; 108(6):492-98.

25. Zaki ME, El-Bassyouni HT, El-Gammal M, Kamal S. Indicators of the metabolic syndrome in obese adolescents. Arch Med Sci. [Internet] 2015; 11, 1: 92-98. Disponible en: http://doi: 10.5114/aoms.2015.49214.

26. Fernández D, Cabrera A, Sanz H, Elosua R, Guembe MJ, Alzamora M, et al. Síndrome Metabólico en España: prevalencia y riesgo coronario asociado a la definición armonizada y a la propuesta de la OMS. Estudio DARIOS. Rev Esp Cardiol. 2012; 65(3): 241-48

27. Ortiz MS, Myers HF, Dunkel -Schetter C, Rodriguez CJ, Seeman TE. Psychosocial predictors of metabolic syndrome among Latino groups in the multi-ethnic study of atherosclerosis (MESA). PLoS One. 2015 [Internet] 2015 Apr 23; 10(4): e0124517. Disponible en: http://doi: 10.1371/journal.pone.0124517.

28. Cruz IRD, Mourão DM, Freitas DA, Silva AG, Ribeiro A, Aidar FJ, et al. Nutritional Status Associated with Metabolic Syndrome in MiddleSchool Children in the City of Montes Claros - MG, Brazil. Journal of Human Kinetics. [Internet] 2014; 43:97-104. Disponible en: http:// doi:10.2478/hukin-2014-0094.

29. Liu X, Tao L, Cao K, Wang Z, Chen D, Guo J, et al. Association of High density lipoprotein with development of metabolic syndrome components: a five year follow up in adults. BMC Public Health.[Internet] 2015 [Citado em 27 de abril de 2015]; 15:412. Disponible en: http:// doi:10.1186/s12889-015-1747-9.

30. Bae HK, Choi HS, Sohn S, Shin H-J, Nam J-H, Hong YM. Cardiovascular Screening in Asymptomatic Adolescents with Metabolic Syndrome.J Cardiovasc Ultrasound. 2015; 23 (1):10-19. doi:10.4250/jcu.2015.23.1.10.

31. Alayón AN, Alvear-Sedan C. Prevalencia de desórdenes del metabolismo de los glúcidos y perfil del diabético en Cartagena de Indias (Colombia), 2005. Salud Uninorte. 2006; 22(1): 20-28. 